\title{
Calcifications in diffuse leptomeningeal glioneuronal tumors: a case series
}

\author{
Giacomo Rebella ${ }^{1}$, Claudia Milanaccio ${ }^{2}$, Giovanni Morana ${ }^{3}$, Domenico Tortora ${ }^{3}$, Antonio Verrico ${ }^{2}$, \\ Gianluca Piatelli ${ }^{4}$, Gabriele Gaggero ${ }^{5}$, Paolo Nozza ${ }^{5}$, Maria Luisa Garrè ${ }^{2}$, Andrea Rossi ${ }^{3}$, Mariasavina \\ Severino $^{3}$
}

${ }^{1}$ Department of Neuroradiology, IRCCS Ospedale Policlinico San Martino, Genova, Italy; ${ }^{2}$ Neuro-oncology Unit, IRCCS Istituto Giannina Gaslini, Genova, Italy; ${ }^{3}$ Neuroradiology Unit, IRCCS Istituto Giannina Gaslini, Genova, Italy; ${ }^{4}$ Neurosurgery Unit, IRCCS Istituto Giannina Gaslini, Genova, Italy; ${ }^{5}$ Pathology Unit, IRCCS Ospedale Policlinico San Martino, Genova, Italy

Correspondence to: Mariasavina Severino. Neuroradiology Unit, IRCCS Istituto Giannina Gaslini, Genova, Italy. Email: mariasavinaseverino@gaslini.org.

\begin{abstract}
Diffuse leptomeningeal glioneuronal tumor (DLGNT) is a new rare entity, typically seen in the pediatric population. Classical neuroimaging features at clinical onset include marked subarachnoid/ leptomeningeal enhancement and tiny pseudo-cystic lesions along the subpial surface of the neuroaxis, frequently associated with communicating hydrocephalus. However, data on the long-term appearance of this tumor on computed tomography (CT) and magnetic resonance imaging (MRI) are still lacking. We describe a peculiar pattern of progressive leptomeningeal calcifications in three young patients with DLGNT. The calcifications, mainly located in the basal cisterns, sylvian fissures and posterior surface of the thalami, were present at clinical onset in the older subject and appeared about 2 years after clinical onset in the other two. Patients underwent different schemes of chemotherapy, variably associated with craniospinal irradiation and/or bevacizumab. In all cases, calcifications were present before starting craniospinal irradiation and/ or treatment with bevacizumab. This novel peculiar pattern of progressive leptomeningeal calcifications expands the imaging phenotype of DLGNT and should be carefully sought, especially in later phases of the disease. Taking into consideration the onset, evolution, and absence of direct relationship with treatments, we hypothesize that calcifications in DLGNTs might be the effect of natural changes in the tumor and its environment.
\end{abstract}

Keywords: Diffuse leptomeningeal glioneuronal tumor (DLGNT); calcifications; radiotherapy; chemotherapy; case report

Submitted Jul 23, 2021. Accepted for publication Jan 25, 2022.

doi: 10.21037/qims-21-741

View this article at: https://dx.doi.org/10.21037/qims-21-741

\section{Introduction}

Diffuse leptomeningeal glioneuronal tumor (DLGNT) is a new entity recognized in the 2016 revision of the World Health Organization (WHO) classification of tumors of the central nervous system $(1,2)$. Previously known in the literature under a variety of similar terms (such as disseminated oligodendroglial-like leptomeningeal tumor of childhood or diffuse leptomeningeal oligodendrogliomatosis) (3-5), this tumor is a rare entity typically seen in the pediatric population with a median age of 8.4 years (6). The neoplastic cells frequently show histological and immunohistochemical features of both glial, defined oligodendroglioma-like cells, and neuronal differentiation, often with expression of synaptophysin in addition to OLIG2 and S-100 (2-4,6-8). The lesions commonly harbor BRAF fusions (KIAA1549-10 BRAF fusion) $(3,4)$ or duplications $(9)$, as well as deletions of chromosome arm $1 \mathrm{p}$, either alone or 11 occasionally combined with $19 \mathrm{q}(3,4,7,10,11)$. In contrast to 

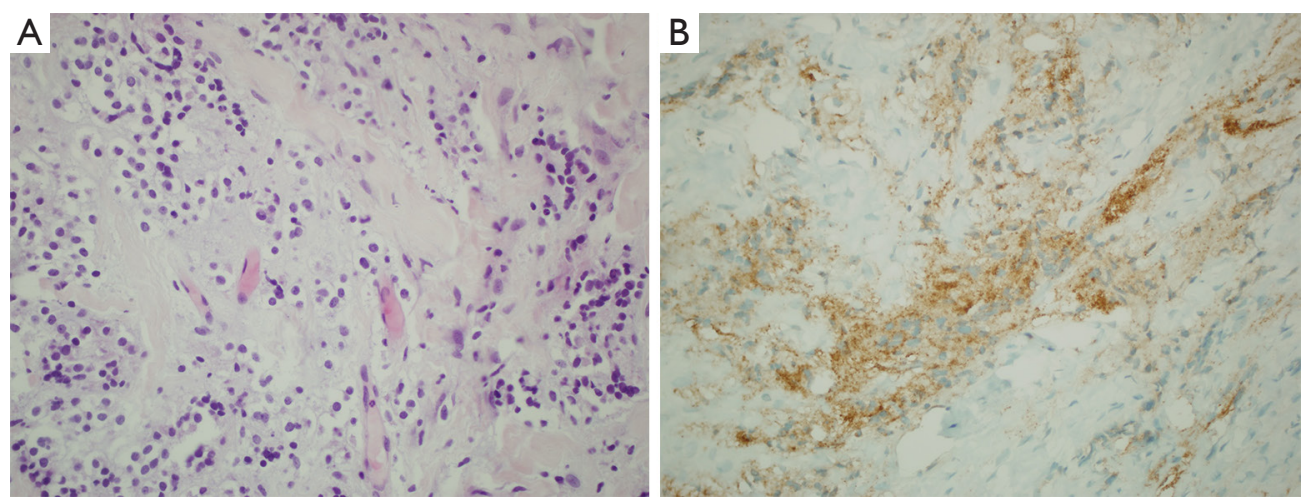

Figure 1 Histologic features in DLGNT. The histological slides of the pathological tissue at the spinal level (Appendix 1, case 3) are obtained after fixation in formalin and inclusion in paraffin. (A) The microscopic examination shows, in the sections stained with hematoxylin-eosin (40x magnification), a moderately cellulated neoplasm consisting of elements embedded in a fibrous stroma (referable to dural tissue) that appear monomorphic, with a rounded/oval profile, with a perinuclear "oligodendroglioma-like" halo, uniform nuclei and an inconspicuous nucleolus. No mitotic figures, microvascular proliferation or necrosis are present; (B) these elements are positive on immunohistochemical characterization for Synaptophysin $(\times 40)$. DLGNT, diffuse leptomeningeal glioneuronal tumors.

oligodendrogliomas, DLGNT do not have IDH mutations $(2,3,6,7,12)$.

Although most tumors display a slow progression, aggressive cases have been occasionally described $(3,5,13,14)$. To date there are no therapeutic guidelines for this rare entity. Published reports cite the use of various chemotherapy regimens with or without radiation, showing relatively good clinical outcomes with adequate disease control in most cases $(2,3,5,6,8,9,15)$.

Nonetheless, survival varies widely and data on longterm outcome are still lacking. Some authors reported an average survival of 22 months (12), while other series showed a range of survival that ranged from 19 months to 9 years, with a mean follow-up time of 54 months (5).

The classical magnetic resonance imaging (MRI) appearance of these tumors consists of marked subarachnoid/leptomeningeal enhancement and tiny T2hyperintense pseudo-cystic lesions along the subpial surface of the neuroaxis, especially in the cerebellum, brainstem, and spinal cord (2,16,17-19). Communicating hydrocephalus is frequently associated (6). Although, by definition, DLGNT should not display significant parenchymal involvement and/or previous parenchymal tumor, few subjects presented an intraparenchymal lesion component, commonly in the spinal cord $(12,20)$. Furthermore, in a recent study by Chiang et al. comprising five patients, leptomeningeal dissemination was not radiologically detected $(1,21)$, suggesting that DLGNT do not necessarily present with gross leptomeningeal dissemination on MRI $(21,22)$.
Here, we report a novel neuroradiological feature, consisting of a peculiar pattern of progressive leptomeningeal calcifications in three young subjects with DLGNT with long-term clinical and neuroradiological follow-up.

We present the following article in accordance with the CARE reporting checklist (available at https://qims. amegroups.com/article/view/10.21037/qims-21-741/rc).

\section{Case presentation}

Three patients with histologically proven DLGNT (2 males and 1 female, median age 12.4 years, range, 9.4-25.8 years) were identified from the institutional database of pediatric patients treated for tumors at the Gaslini Children's Hospital between 2010 and 2020. The median age at diagnosis was 5 years (range, 2-13 years) and mean followup time was 9.5 years (range, $8.4-12.8$ years). In all our cases an immunohistochemical study of the biopsied pathological tissue using antibodies for IDH1 (R132H), GFAP, Olig2, S100 and synaptophysin was performed, detecting the following pattern: IDH1 negative, GFAP negative, OLIG2 positive, S100 positive, synaptophysin positive (Figure 1).

In $2 / 3$ cases clinical presentation at onset included photophobia and vomiting related to a communicating hydrocephalus which was confirmed by brain CT or MRI examinations while the third case had symptoms more nuanced and non-specific, characterized by awakening at night, confusional state, and dysarthria. All patients were evaluated with brain and spinal MRI that in $2 / 3$ cases 

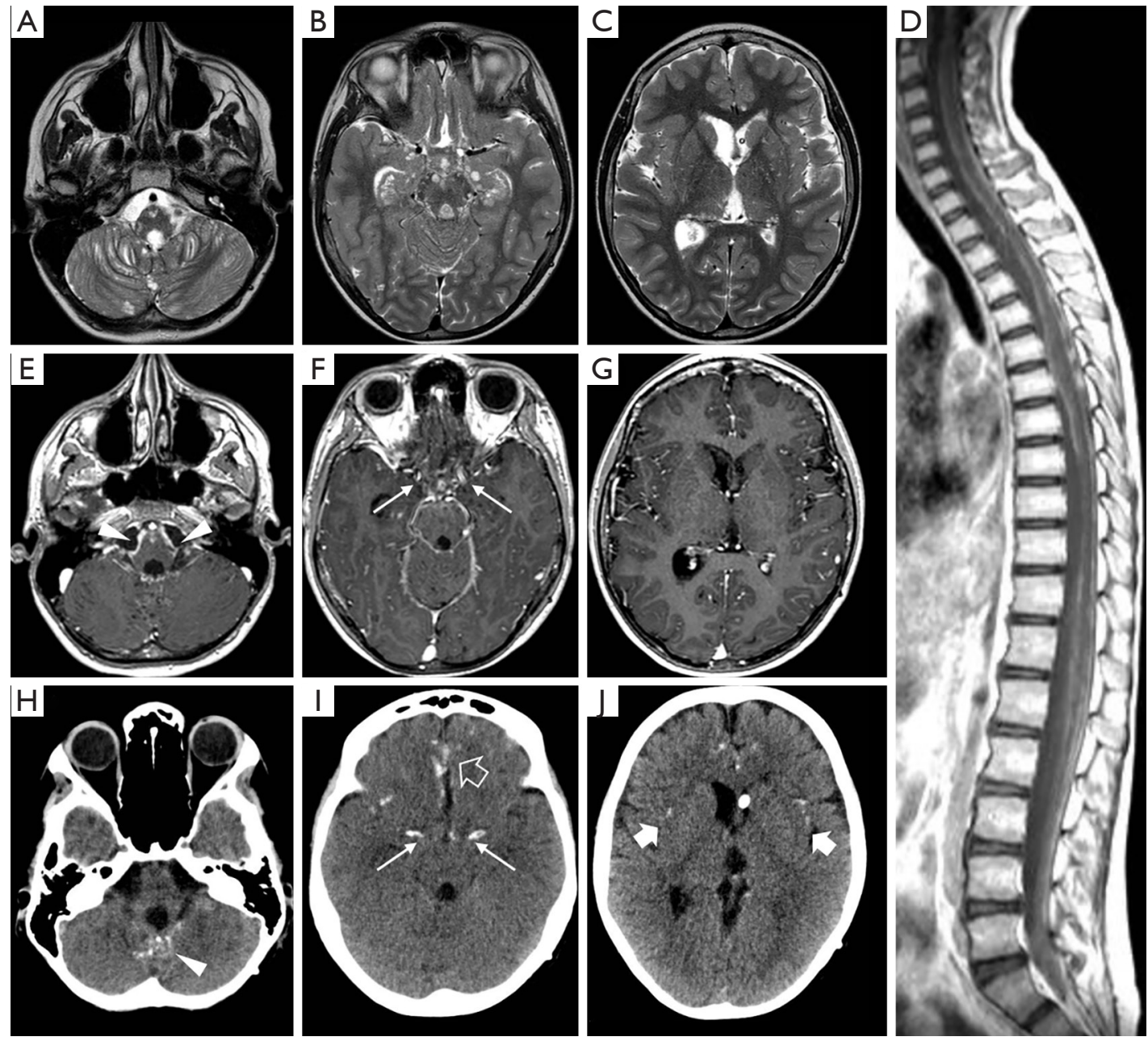

Figure 2 Brain and spinal MRI and CT scans of patient \#1 performed at last follow-up at the age of 12.4 years. Axial T2-weighted (A-C) images show multiple small cysts scattered on the surface of the cerebellum, brainstem, mesial temporal lobes, and hypothalamus. Postcontrast T1-weighted images (D-G) reveal contrast enhancement along the surface of the spinal cord, roots of the cauda equina, and brainstem (arrowheads), and at the level of basal cisterns (arrows). Brain CT images (H-J) reveal leptomeningeal calcifications at the level of inferior cerebellar vermis (arrowhead), basal cisterns (arrows), frontal (empty arrow) and insular sulci (thick arrows). MRI, magnetic resonance imaging; CT, computed tomography.

showed multiple small cysts scattered on the surface of cerebellum, brainstem, and spinal cord (Figure $2 A-2 C$ ); in the older subject, small cystic lesions were present only on the cerebellar surface. In all cases, contrast-enhanced T1-weighted images revealed diffuse leptomeningeal enhancement mainly at level of cerebellar subarachnoid spaces, basal cisterns, and spinal cord with no evidence of intraparenchymal involvement (Figure 2D-2G).

Calcifications were present at clinical onset in the older subject and appeared about 2 years after clinical onset in the other two. They were mainly located in the basal cisterns, sylvian fissures, on the posterior surface of the thalami, and within cerebral/cerebellar hemispheric sulci (Figure 2H-27). At first, calcifications were mostly visible as slight hypointensities on $\mathrm{T} 2{ }^{*}$ gradient echo (GE) or susceptibility weighted images (SWI) corresponding to subtle hyperdensities on CT scan. Over time, multiple MRI and CT examinations showed reduction of the leptomeningeal enhancement and a progressive increase in signal and density alterations referable to calcifications (Figure 3). Figure 4 shows progression of calcifications on multiple head CT examinations over time in each patient. 

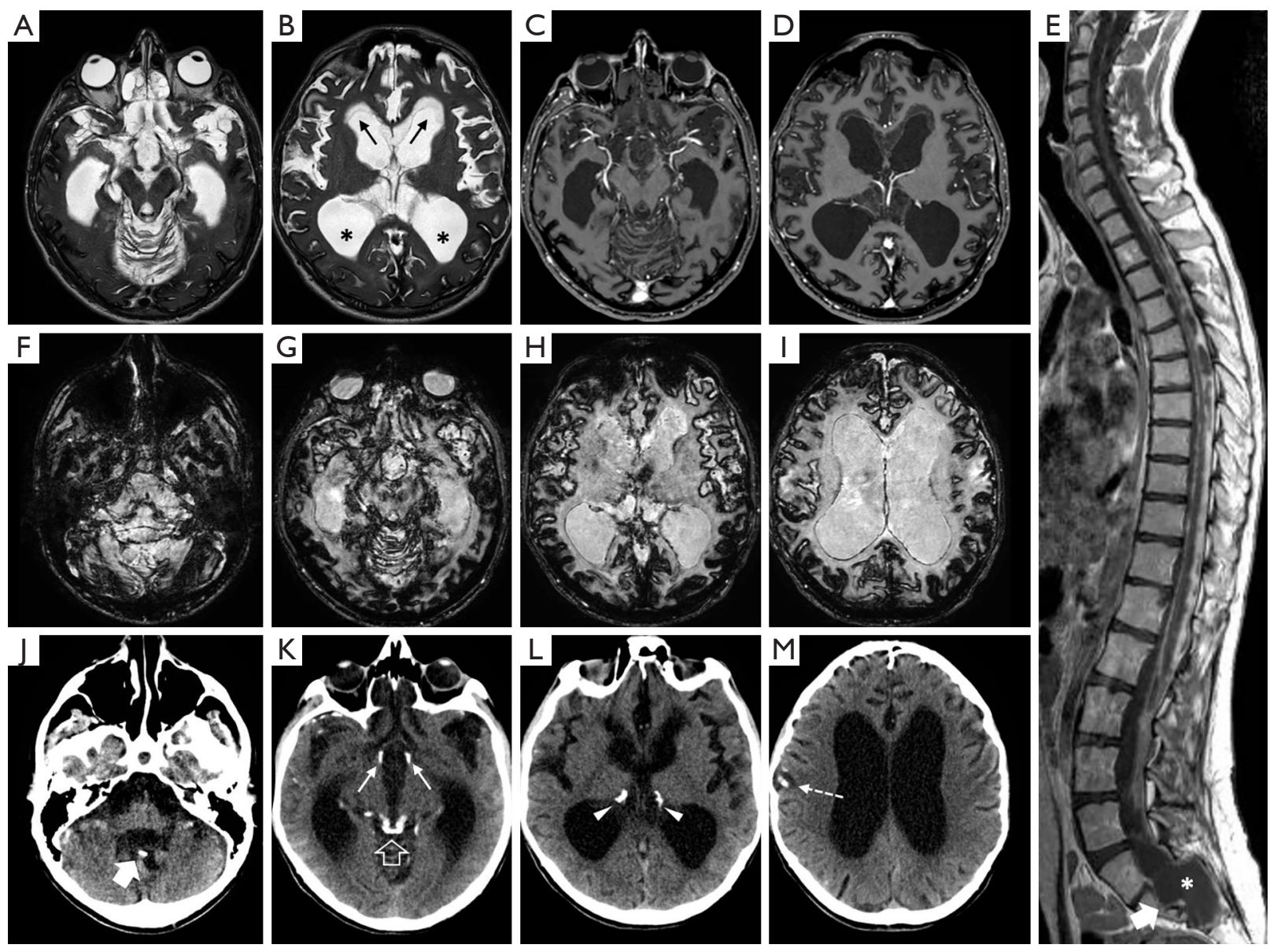

Figure 3 Brain and spinal MRI and CT scans of patient \#3 performed at last follow-up at the age of 25 years. Axial T2-weighted (A, B) and post-contrast T1-weighted (C-E) images show markedly enlarged cerebral and cerebellar subarachnoid spaces with confluent non-enhancing cystic changes, also visible along the frontal horns of the lateral ventricles (arrows). The periventricular white matter is markedly thinned with consequent enlargement of the lateral ventricles (asterisks). At this stage, there is no contrast-enhancement of the leptomeninges around the brain, but the cortex appears diffusely hypointense on both T1- and T2-weighted images. Sagittal contrast-enhanced T1weighted image of the spine (E) demonstrates diffuse enhancement along the surface of the spinal cord and cauda equina roots, associated with marked enlargement of the subarachnoid spaces (asterisk) and bone scalloping (thick arrow) at the level of the dural cul-the-sac (this feature was not present at clinical onset); (F-I) axial SWI reveals marked hypointensities involving the cerebral and cerebellar cortex and the leptomeninges surrounding the brainstem. A faint linear hypointensity is also noted along the ventricular ependyma; (J-M) corresponding CT images show multiple coarse nodular calcifications at the level of the inferior vermis (thick arrow), hypothalamus (arrows), posterior midbrain (empty arrow) and mesial thalami (arrowheads), and along some sulci in the right frontal region (dotted arrow). MRI, magnetic resonance imaging; CT, computed tomography; SWI, susceptibility weighted imaging.

Patients underwent different schemes of chemotherapy, variably associated with craniospinal irradiation and/or bevacizumab, with disease stabilization in one case, disease regression but demise after bilateral cerebrovascular accidents and radiation induced leukoencephalopathy in the second case (Figure 5), and disease progression in the third case. In all cases, calcifications were present before starting craniospinal irradiation and/or treatment with bevacizumab; in one of the three cases subtle calcifications were already present at diagnosis.

Clinical and neuroradiological features of each patient are described in detail as Supplementary file (Appendix 1). All procedures performed in this study were in accordance with the ethical standards of the institutional and/or national 

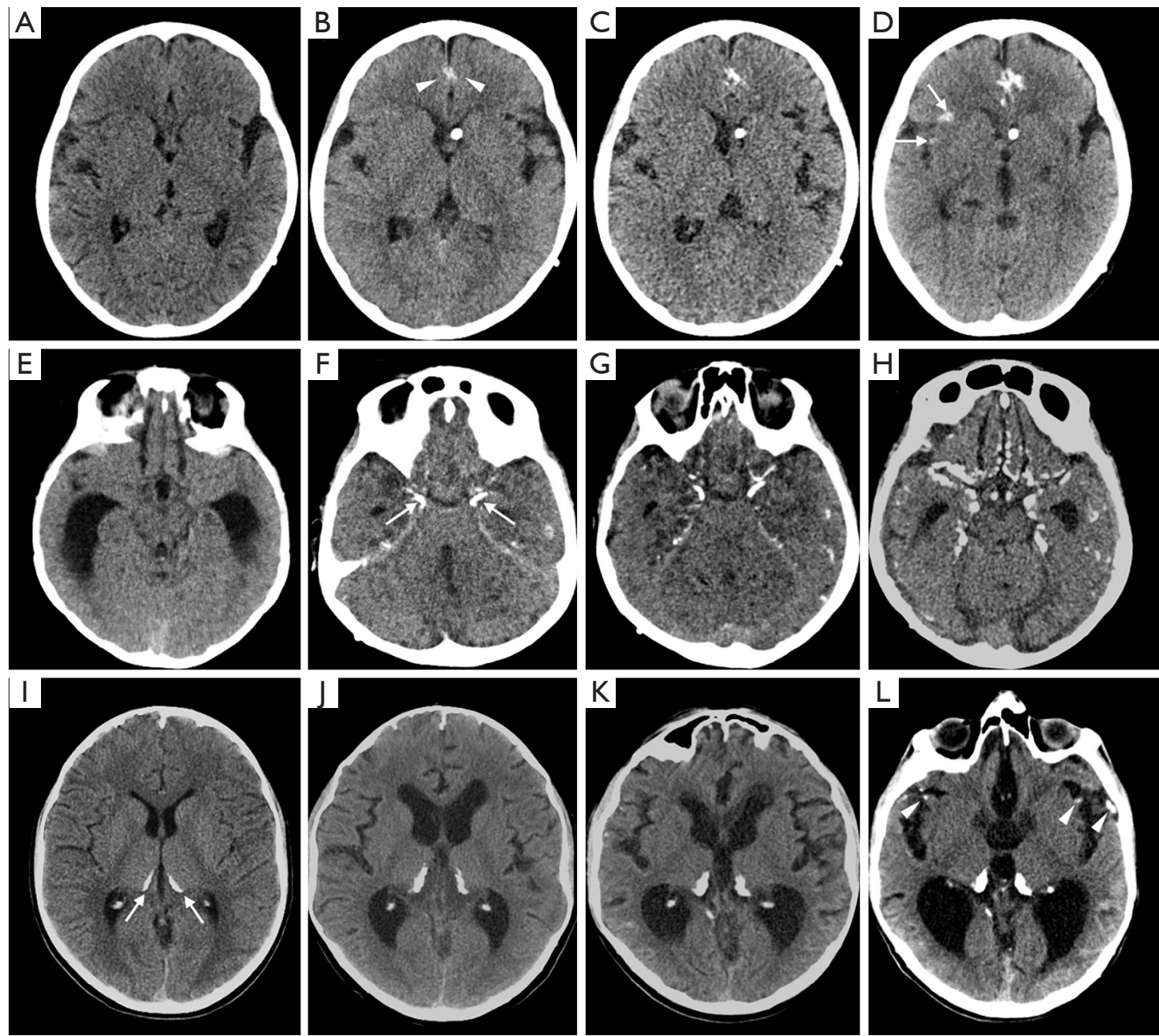

Figure 4 Head CT examinations showing the evolution of intracranial calcifications in patients with DLGNT. (A-D) patient \#1: (A) CT at clinical onset (4 years of age) after ventricular shunting reveals no intracranial calcifications; (B) after 3 years, at the age of 7 years, subtle leptomeningeal calcifications appear at the level of anterior mesial frontal gyri (arrowheads), and progressively increase at subsequent CT examinations performed at 9 years (C) and at 11 years of age (D). New calcifications are visible at the level of right insula (arrows); (E-H) patient \#2: (E) CT at clinical onset (2 years of age) demonstrates a communicating hydrocephalus without intracranial calcifications; (F) after 2 years, at the age of 4 years, multiple calcifications are noted at the level of basal cisterns (arrows), that progressively increase in number and size at subsequent examinations performed at 6 years $(\mathrm{G})$ and 9 years $(\mathrm{H})$, also involving the basal frontal and mesial temporal sulci, the cerebral convexity and hypothalamus; (I-L) patient \#3: (I) CT at clinical onset (13 years of age) shows presence of calcifications along the posteromedial surface of the thalami (arrows). Follow-up CT scans performed at 15 years (J), 20 years $(\mathrm{K})$ and 25 years of age (L) demonstrate increase of calcifications at the level of the leptomeninges over the posteromedial surfaces of the thalami and scattered along the cerebral convex sulci (arrowheads). Note the progressive enlargement of the lateral ventricles and subarachnoid spaces. DLGNT, diffuse leptomeningeal glioneuronal tumors; CT, computed tomography.

research committee(s) and with the Helsinki Declaration (as revised in 2013). Written informed consent was obtained from the patients' parents or legal guardians for publication of this case report and accompanying images. A copy of the written consent is available for review by the editorial office of this journal. 

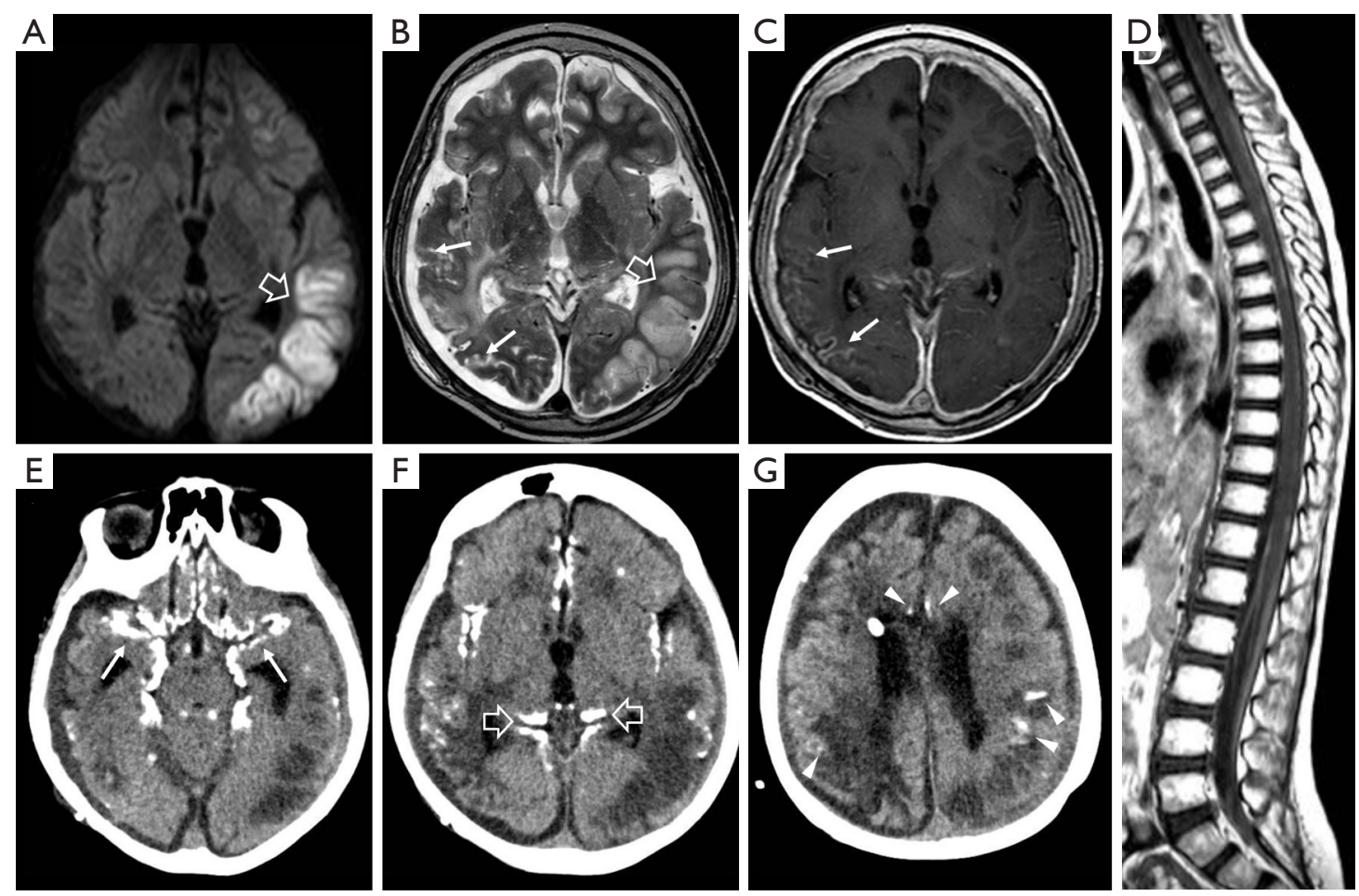

Figure 5 Brain and spinal MRI and CT scans of patient \#2 performed at last follow-up at the age of 9 years. Axial diffusion-weighted (A), T2-weighted (B) and post-contrast T1-weighted (C) images reveal an acute ischemic infarct at the level of the left temporal corticosubcortical region (empty arrows), in the posterior peripheral branches of the middle cerebral artery, associated with cortico-subcortical gliosis and atrophy with laminar necrosis at the level of the contralateral temporal lobe (thin arrows), resulting from the previous ischemic infarct in the right middle cerebral artery territory. There are also diffuse deep white matter signal changes, likely related to radiationinduced leukoencephalopathy. Note the enlargement of subarachnoid spaces and thickening and contrast enhancement of the dura overlying the cerebral convexity; (D) sagittal contrast-enhanced T1-weighted image of the spine demonstrates diffuse enhancement along the surface of the spinal cord as well as the roots of the cauda equina; (E-G) axial head CT images show widespread confluent calcifications at the level of basal cisterns (arrows) and hypothalamus, and along the surface of the mesial temporal, frontal, and occipital lobes, insulae and pulvinar (empty arrows). Few nodular calcifications are also scattered in the depth of fronto-temporo-parietal sulci (arrowheads). MRI, magnetic resonance imaging; CT, computed tomography.

\section{Discussion}

In this case series, we report a peculiar pattern of intracranial calcifications detected on MRI and CT studies in three patients with typical radiological features and histological diagnosis of DLGNT.

The localization of calcium deposits was similar in all cases and corresponded to the regions classically involved by leptomeningeal contrast enhancement in DLGNT. In particular, calcifications were mainly located in the basal cisterns, sylvian fissures, on the posterior surface of the thalami, and within cerebral hemispheric sulci. We did not perform T2* GE sequences or CT scan at the spinal level. Therefore, we are unaware if calcifications may also be present around the spinal cord, along the spinal roots or at the level of neoplastic tissue in thecal sac, where the biopsies were performed. Interestingly, few nodular calcifications were recently noted by Tiwari et al. along the left sylvian fissure in a 3-year-old girl with a DLGNT on a head CT at clinical onset (23). Unfortunately, in this case, the biopsy was performed along the contralateral sylvian fissure, and histopathological correlation is unavailable also in this case (23). Recently, Lakhani et al. described a case of DLGNT with an intracranial calcified parenchymal mass lesion in the right basal ganglia at the onset (24); in this case, the coarse intraparenchymal calcific deposit seems to differ from those illustrated in our cases in which the calcifications turn out to be a peculiar pattern 
of leptomeningeal calcifications which seem to be a characteristic of this neoplasm if followed over time. These types of calcifications are different from those we might find in other primary central nervous system tumors that present as partly calcified intraparenchymal masses such as oligodendroglioma or calcifying pseudoneoplasms of neuroaxis (CAPNON), which usually occur as extra- axial, or less often intra-axial, solitary calcified masses.

Of note, in the rare cases reported in literature in which a histopathologic evaluation of DLGNT was performed during long-term follow-up or at autopsy, no calcium deposits have been described $(3,25)$. Histologically, DLGNT are described as low- to moderate-cellularity lesions consisting of relatively monomorphous oligodendrocyte-like cells with round to oval nuclei, inconspicuous nucleoli and glioneuronal elements, embedded in a plentiful desmoplastic or myxoid leptomeningeal stroma $(1-3,5,22)$. Progressive deposition of calcium in the context of this desmoplastic tissue might be a possible explanation for leptomeningeal calcifications in DLGNT. Indeed, calcifications have been described in other glioneuronal tumors, particularly solid or solid-cystic tumors like ganglioglioma (30\%), desmoplastic infantile ganglioglioma (20\%), papillary glioneuronal tumors $(75 \%)$ and rosette-forming glioneuronal tumor (25\%) (26-28).

Since the first report by Harwood-Nash et al. (29), calcifications are considered the most common radiological manifestations of radiation-related brain injury, though the underlying mechanism is not yet completely clear. It has been suggested that post-radiation intracerebral calcifications could be related to mineralizing microangiopathy or dystrophic calcifications developed in the perivascular brain tissue secondary to the leakage of plasma fluid from damaged vessels, and regional ischemia due to microcirculation impairment (30). However, in the present series, only one subject was treated with radiotherapy, and intracranial calcifications appeared well before the onset of radiation treatment. Of note, in this patient, we observed a dramatic evolution with radiation induced leukoencephalopathy associated with right middle cerebral artery infarct that could have been triggered or facilitated by presence of calcifications around the arterial walls in the basal cisterns.

Systemic chemotherapy could also induce the formation of calcifications within tumors. It has been hypothesized that chemotherapy may lead to a $\mathrm{pH}$ rise with alkalization of the tumor environment and subsequent degeneration of the neoplastic tissues followed by calcifications of necrotic components (31). However, there is still no absolute certainty about the mechanism and no specific treatment has been clearly indicated as responsible. Indeed, the appearance of calcifications has not been systematically reported in patients with low-grade brain tumors treated with International Society of Paediatric Oncology-Low Grade Glioma Subcommittee (SIOP-LGG) chemotherapy regimens. Therefore, we believe that calcifications in DLGNT are not related to radiotherapy and are unlikely the consequence of chemotherapy. Of note, two patients in this series were treated with bevacizumab. Bevacizumab is a humanized monoclonal antibody that works by inhibiting the action of VEGF, a specific angiogenesis growth factor that binds to receptors on blood vessels and stimulates the formation of new blood vessels.

Bevacizumab is the prototype of an antiangiogenic agent and has broad antitumor activity. It has been used in DLGNT, individually or in combination with radiotherapy, chemotherapy and/or other targeted therapies, with mixed efficacy $(4,9,13,14,32,33)$. Bevacizumab may cause intratumoral calcifications in high-grade gliomas, likely through a vascular mechanism (34). It has been hypothesized that bevacizumab can lead to hypoxia of the neoplastic tissue with consequent breakdown of the normal calcium gradient across the cell membrane and accumulation of calcium inside the cell and in the extracellular space after cell death $(31,33)$. Of note, in the two patients treated with bevacizumab, calcifications were already present before the beginning of this therapy. Therefore, we suppose that bevacizumab might perhaps have had a contributory effect but not a primary role in the development of leptomeningeal calcium deposits.

Interestingly, in our series, the number and extension of calcifications progressively increased, starting from few linear/nodular leptomeningeal depositions and slowly progressing to a more diffuse pattern of leptomeningeal involvement. At the onset, although calcifications could be depicted on brain MRI as faint, subtle hypointensities on $\mathrm{T} 2{ }^{*} \mathrm{GE}$ or susceptibility-weighted images, these were better appreciated as linear or nodular leptomeningeal hyperdensities on head CT examinations. Conversely, in one subject (Appendix 1, case 3), several years after clinical onset and before bevacizumab therapy, we noticed a discrepancy between the evolution of these imaging features on CT and MRI studies. Indeed, leptomeningeal calcifications on head CT were less extensive and conspicuous compared to the linear hypointensities depicted on $\mathrm{T} 2{ }^{*} \mathrm{GE}$ or susceptibility-weighted sequences on brain MRI. Moreover, 
these hypointensities also involved the cortex of both cerebral hemispheres. This finding suggests that cortical neurodegenerative processes might be associated with leptomeningeal calcium deposits in DLGNT in later stages. Remarkably, the patient presented signs of cognitive impairment supporting a neurodegenerative process. However, we lack cortical histological data, and the nature of these T2 $^{*}$-hypointense cortical lesions in DLGNT remains unknown.

In conclusion, we reported a novel peculiar pattern of progressive leptomeningeal calcifications in DLGNT, thus expanding the imaging phenotype of this rare neoplasm. Taking into consideration the onset, evolution of this neuroimaging feature and the absence of direct relationship with treatments, we hypothesize that calcifications in DLGNT might be the effect of natural changes in the tumor and its environment. However, larger longitudinal studies with histopathological correlation are needed to understand the real nature of this neuroimaging finding, the underlying mechanisms, and the potential contributing role of oncology treatment.

\section{Acknowledgments}

We acknowledge all the members of the pediatric brain tumors multidisciplinary team of the IRCCS Istituto Giannina Gaslini, who were involved in the care of these patients.

Funding: This study was funded by from "Associazione per la Ricerca sui Tumori Cerebrali del Bambino" and from "Fondazione Berlucchi" to MLG (Neuroncology Unit, IRCCS Istituto Giannina Gaslini).

\section{Footnote}

Reporting Checklist: The authors have completed the CARE reporting checklist. Available at https://qims.amegroups. com/article/view/10.21037/qims-21-741/rc

Conflicts of Interest: All authors have completed the ICMJE uniform disclosure form (available at https://qims. amegroups.com/article/view/10.21037/qims-21-741/coif). The authors have no conflicts of interest to declare.

Ethical Statement: The authors are accountable for all aspects of the work in ensuring that questions related to the accuracy or integrity of any part of the work are appropriately investigated and resolved. All procedures performed in this study were in accordance with the ethical standards of the institutional and/or national research committee(s) and with the Helsinki Declaration (as revised in 2013). Written informed consent was obtained from the patients' parents or legal guardians for publication of this case report and accompanying images. A copy of the written consent is available for review by the editorial office of this journal.

Open Access Statement: This is an Open Access article distributed in accordance with the Creative Commons Attribution-NonCommercial-NoDerivs 4.0 International License (CC BY-NC-ND 4.0), which permits the noncommercial replication and distribution of the article with the strict proviso that no changes or edits are made and the original work is properly cited (including links to both the formal publication through the relevant DOI and the license). See: https://creativecommons.org/licenses/by-nc-nd/4.0/.

\section{References}

1. Louis DN, Perry A, Wesseling P, Brat DJ, Cree IA, Figarella-Branger D, Hawkins C, Ng HK, Pfister SM, Reifenberger G, Soffietti R, von Deimling A, Ellison DW. The $2021 \mathrm{WHO}$ classification of tumors of the central nervous system: a summary. Neuro Oncol 2021;23:1231-51.

2. Gardiman MP, Fassan M, Orvieto E, D'Avella D, Denaro L, Calderone M, Severino M, Scarsello G, Viscardi E, Perilongo G. Diffuse leptomeningeal glioneuronal tumors: a new entity? Brain Pathol 2010;20:361-6.

3. Rodriguez FJ, Perry A, Rosenblum MK, Krawitz S, Cohen KJ, Lin D, Mosier S, Lin MT, Eberhart CG, Burger PC. Disseminated oligodendroglial-like leptomeningeal tumor of childhood: a distinctive clinicopathologic entity. Acta Neuropathol 2012;124:627-41.

4. Rodriguez FJ, Schniederjan MJ, Nicolaides T, Tihan T, Burger PC, Perry A. High rate of concurrent BRAFKIAA1549 gene fusion and $1 \mathrm{p}$ deletion in disseminated oligodendroglioma-like leptomeningeal neoplasms (DOLN). Acta Neuropathol 2015;129:609-10.

5. Preuss M, Christiansen H, Merkenschlager A, Hirsch FW, Kiess W, Müller W, Kästner S, Henssler A, Pekrun A, Hauch H, Nathrath M, Meixensberger J, Pietsch T, Kuchelmeister K. Disseminated oligodendrogliallike leptomeningeal tumors: preliminary diagnostic and therapeutic results for a novel tumor entity corrected. J Neurooncol 2015;124:65-74.

6. Chen W, Kong Z, Fu J, Zhao D, Wang R, Ma W, Wang Y. 
Diffuse leptomeningeal glioneuronal tumour (DLGNT) with hydrocephalus as an initial symptom: a case-based update. Childs Nerv Syst 2020;36:459-68.

7. Schniederjan MJ, Alghamdi S, Castellano-Sanchez A, Mazewski C, Brahma B, Brat DJ, Brathwaite CD, Janss AJ. Diffuse leptomeningeal neuroepithelial tumor: 9 pediatric cases with chromosome $1 \mathrm{p} / 19 \mathrm{q}$ deletion status and IDH1 (R132H) immunohistochemistry. Am J Surg Pathol 2013;37:763-71.

8. Aguilera D, Castellino RC, Janss A, Schniederjan M, McNall R, MacDonald T, Mazewski C. Clinical responses of patients with diffuse leptomeningeal glioneuronal tumors to chemotherapy. Childs Nerv Syst 2018;34:329-34.

9. Dodgshun AJ, SantaCruz N, Hwang J, Ramkissoon SH, Malkin H, Bergthold G, Manley P, Chi S, MacGregor D, Goumnerova L, Sullivan M, Ligon K, Beroukhim R, Herrington B, Kieran MW, Hansford JR, Bandopadhayay P. Disseminated glioneuronal tumors occurring in childhood: treatment outcomes and BRAF alterations including V600E mutation. J Neurooncol 2016;128:293-302.

10. Deng MY, Sill M, Chiang J, Schittenhelm J, Ebinger M, Schuhmann MU, et al. Molecularly defined diffuse leptomeningeal glioneuronal tumor (DLGNT) comprises two subgroups with distinct clinical and genetic features. Acta Neuropathol 2018;136:239-53.

11. Nambirajan A, Suri V, Kedia S, Goyal K, Malgulwar PB, Khanna G, Panda PK, Gulati S, Garg A, Sharma MC. Paediatric diffuse leptomeningeal tumor with glial and neuronal differentiation harbouring chromosome $1 \mathrm{p} / 19 \mathrm{q}$ co-deletion and H3.3 K27M mutation: unusual molecular profile and its therapeutic implications. Brain Tumor Pathol 2018;35:186-91.

12. Cho HJ, Myung JK, Kim H, Park CK, Kim SK, Chung CK, Choi SH, Park SH. Primary diffuse leptomeningeal glioneuronal tumors. Brain Tumor Pathol 2015;32:49-55.

13. Yamasaki T, Sakai N, Shinmura K, Kawaji H, Koizumi S, Samashima T, Namba H. Anaplastic changes of diffuse leptomeningeal glioneuronal tumor with polar spongioblastoma pattern. Brain Tumor Pathol 2018;35:209-16.

14. Kessler BA, Bookhout C, Jaikumar S, Hipps J, Lee YZ. Disseminated oligodendroglial-like leptomeningeal tumor with anaplastic progression and presumed extraneural disease: case report. Clin Imaging 2015;39:300-4.

15. Lyle MR, Dolia JN, Fratkin J, Nichols TA, Herrington BL. Newly identified characteristics and suggestions for diagnosis and treatment of diffuse leptomeningeal glioneuronal/neuroepithelial tumors: a case report and review of the literature. Child Neurol Open 2015;2:2329048X14567531.

16. Karlowee V, Kolakshyapati M, Amatya VJ, Takayasu T, Nosaka R, Sugiyama K, Kurisu K, Yamasaki F. Diffuse leptomeningeal glioneuronal tumor (DLGNT) mimicking Whipple's disease: a case report and literature review. Childs Nerv Syst 2017;33:1411-4.

17. Lee JK, Ko HC, Choi JG, Lee YS, Son BC. A case of diffuse leptomeningeal glioneuronal tumor misdiagnosed as chronic tuberculous meningitis without brain biopsy. Case Rep Neurol Med 2018;2018:1391943.

18. Ghoniya R, Raheja A. Imaging in diffuse leptomeningeal glioneuronal tumor. Neurol India 2019;67:615-6.

19. Demir MK, Yapıcıer O, Yılmaz B, Kılıç T. Magnetic resonance imaging findings of mixed neuronal-glial tumors with pathologic correlation: a review. Acta Neurol Belg 2018;118:379-86.

20. Xu H, Chen F, Zhu H, Luo L, Zhang R. Diffuse leptomeningeal glioneuronal tumor in a Chinese adult: a novel case report and review of literature. Acta Neurol Belg 2020;120:247-56.

21. Chiang JCH, Harreld JH, Orr BA, Sharma S, Ismail A, Segura AD, Ellison DW. Low-grade spinal glioneuronal tumors with BRAF gene fusion and $1 \mathrm{p}$ deletion but without leptomeningeal dissemination. Acta Neuropathol 2017;134:159-62.

22. Kang JH, Buckley AF, Nagpal S, Fischbein N, Peters KB. A diffuse leptomeningeal glioneuronal tumor without diffuse leptomeningeal involvement: detailed molecular and clinical characterization. J Neuropathol Exp Neurol 2018;77:751-6.

23. Tiwari S, Yadav T, Pamnani J, Mathew JM, Elhence P, Praneeth K, Vedant D, Khera PS, Garg P, Vyas V. Diffuse leptomeningeal glioneuronal tumor: a unique leptomeningeal tumor entity. World Neurosurg 2020;135:297-300.

24. Lakhani DA, Mankad K, Chhabda S, Feizi P, Patel R, Sarma A, Pruthi S. Diffuse Leptomeningeal Glioneuronal Tumor of Childhood. AJNR Am J Neuroradiol 2020;41:2155-9.

25. Gardiman MP, Fassan M, Nozza P, Orvieto E, Garrè ML, Milanaccio C, Severino M, Perilongo G, Giangaspero F. Diffuse leptomeningeal glioneuronal tumours: clinicopathological follow-up. Pathologica 2012;104:428-31.

26. Shin JH, Lee HK, Khang SK, Kim DW, Jeong AK, Ahn KJ, Choi CG, Suh DC. Neuronal tumors of the central nervous system: radiologic findings and pathologic 
correlation. Radiographics 2002;22:1177-89.

27. Yadav N, Rao S, Saini J, Prasad C, Mahadevan A, Sadashiva N. Papillary glioneuronal tumors: a radiopathologic correlation. Eur J Radiol 2017;97:44-52.

28. Hsu C, Kwan G, Lau Q, Bhuta S. Rosette-forming glioneuronal tumour: imaging features, histopathological correlation and a comprehensive review of literature. Br J Neurosurg 2012;26:668-73.

29. Harwood-Nash DC, Reilly BJ. Calcification of the basal ganglia following radiation therapy. Am J Roentgenol Radium Ther Nucl Med 1970;108:392-5.

30. Suzuki S, Nishio S, Takata K, Morioka T, Fukui M. Radiation-induced brain calcification: paradoxical high signal intensity in T1-weighted MR images. Acta Neurochir (Wien) 2000;142:801-4.

31. Halpin S, Kingsley D. Disappearance of cerebral

Cite this article as: Rebella G, Milanaccio C, Morana G, Tortora D, Verrico A, Piatelli G, Gaggero G, Nozza P, Garrè ML, Rossi A, Severino M. Calcifications in diffuse leptomeningeal glioneuronal tumors: a case series. Quant Imaging Med Surg 2022;12(5):2985-2994. doi: 10.21037/qims21-741 calcification as a sign of tumor growth. AJNR Am J

Neuroradiol 1993;14:119-22.

32. Schwetye KE, Kansagra AP, McEachern J, Schmidt RE, Gauvain K, Dahiya S. Unusual high-grade features in pediatric diffuse leptomeningeal glioneuronal tumor: comparison with a typical low-grade example. Hum Pathol 2017;70:105-12.

33. Blumenthal DT, Aisenstein O, Ben-Horin I, Ben Bashat D, Artzi M, Corn BW, Kanner AA, Ram Z, Bokstein F. Calcification in high grade gliomas treated with bevacizumab. J Neurooncol 2015;123:283-8.

34. Pellerino A, Rudà R, Bertero L, Magistrello M, Franchino F, Cassoni P, Pasqualetti F, Pinessi L, Giangaspero F, Soffietti R. Successful use of bevacizumab in an adult primary diffuse leptomeningeal glioneuronal tumor. J Neurosurg Sci 2018;62:229-32. 


\section{Case 1}

A 4-year-old boy presented with photophobia, abdominal pain, vomiting, and anorexia with severe weight loss in the last 2 months. Craniospinal magnetic resonance imaging (MRI) revealed communicating hydrocephalus associated with multiple small cysts scattered on the surface of the cerebellum, brainstem, mesial temporal lobes, and spinal cord. Diffuse gadolinium enhancement involving the leptomeninges surrounding the brain and spinal cord as well as the roots of the cauda equina was noted without evidence of an intraparenchymal mass. Head computed tomography (CT) showed absence of evident intracranial calcifications. The patient underwent ventriculoperitoneal shunt surgery. Infectious cerebrospinal fluid markers were negative. A biopsy of the pathological tissue at the level of the caudal roots was performed and a diagnosis of diffuse leptomeningeal glioneuronal tumor (DLGNT) was reached through anatomopathological and immunohistochemical analysis. The child was treated with systemic chemotherapy, at first with 3 consecutive cycles of high-dose Methotrexate, and subsequently according to a modified International Society of Pediatric Oncology-Low Grade Glioma (SIOPLGG) protocol (Vincristine and Cyclophosphamide alternating with Vincristine and Cisplatin) for 1 year. The response was good with improvement of cachexia and weight recovery. The patient then presented phases of stability alternated with phases of mild progression. About 2 years after diagnosis, craniospinal MRI showed an increase in size and number of the cysts and revealed linear hypointensities on $\mathrm{T}^{*}$ gradient echo (GE) images along some anterior mesial frontal and inferior cerebellar gyri and in the basal cisterns, that corresponded to subtle hyperdensities on CT scan. These signal and density alterations progressively increased in the following years, with clear formation of calcifications at the level of cerebellar vermis, basal cisterns, sylvian fissures and bilateral frontal sulci (Figure 4A-4D). During progression phases, the patient was treated with oral Temozolomide and Etoposide, followed by Vinblastine, Vinorelbine and finally TPCV regimen (Thioguanine, Procarbazine, Lomustine, and Vincristine). Two and a half years after stop therapy, at the age of 10 , the patient presented with occipital pain, stiffness and lack of appetite. Craniospinal MRI showed increase of the leptomeningeal contrast enhancement. Due to both clinical and radiological disease progression, wanting to avoid craniospinal radiotherapy and considering the reported efficacy in an adult with primary diffuse leptomeningeal glioneuronal tumor (34), we decided to treat the patient with Bevacizumab $(10 \mathrm{mg} / \mathrm{Kg} /$ dose biweekly), that was administered for 15 months, with regression of neurological symptoms. Craniospinal MRI studies revealed initial reduction of leptomeningeal contrast enhancement, followed by stabilization of the neuroimaging features, while head CT demonstrated increased leptomeningeal calcifications (Figure 2). At present, the child is 12.4 years old and remains asymptomatic.

\section{Case 2}

A 2-year-old girl was admitted to our institution because of progressive paraparesis, fecal/urinary incontinence, photophobia, vomiting and bilateral clonus. On admission, head CT revealed communicating hydrocephalus without visible intracerebral masses nor calcifications (Figure 4E). Craniospinal MRI showed cystic lesions diffusely scattered throughout the cerebellum, basal cisterns and on the surface of the brainstem. Contrast-enhanced T1-weighted images revealed diffuse marked thickening and enhancement of the leptomeninges, particularly in the basal cisterns, sylvian fissures, interhemispheric fissure, and spinal cord. There were also multiple non-enhancing cystic lesions along the spinal cord associated with focal dilatations of the ependymal canal. This patient was initially diagnosed with tuberculous meningitis despite absence of laboratory evidence. A ventriculoperitoneal shunting was placed and empirical treatment against tuberculosis was started, which proved ineffective. Therefore, a biopsy of the pathological tissue at the level of the cauda roots was performed and a diagnosis of DLGNT was made. The patient was treated with systemic chemotherapy according to the International SIOP-LGG with vincristine and carboplatin for 18 months, with clinical improvement and radiological stability. Craniospinal MRI performed every 3 months revealed progression of the cystic lesions and leptomeningeal enhancement. Moreover, head CT documented subtle calcified depositions in the basal cisterns (Figure 4F). Therefore, 18 months after the end of first line therapy, we treated the patients with oral Temozolomide and Etoposide, without radiological improvement, and we proposed to proceed with craniospinal radiotherapy, but parents strongly refused it. Therefore, we continued chemotherapy with Vinblastine for 6 months, but after an initial radiological stability, the disease continued to progress. Subsequently, the parents asked to be referred for a second opinion to another hospital, where craniospinal tomotherapy (IMRT-Tomo) was administered with clinical 
and radiological improvement. The initial dose was 32.4 Gy in 18 fractions to the craniospinal axis, followed by a first boost of 9 Gy in 5 fractions to a reduced volume of brain and spine, and a second boost of 12.6 Gy in 7 fractions on a further reduced volume involving brain, brainstem, and spinal cord. However, after about 1.5 years, she returned to our institution for the appearance of acute repetitive seizures and left hemiplegia. Head CT demonstrated a hypodensity in the right fronto-temporo-parietal regions, increased calcifications in the basal cisterns, and new widespread calcifications at the level of medial temporal lobes, sylvian fissure, interhemispheric fissure, and frontal-parietal gyri (Figure 4G,4H). Craniospinal MRI revealed a subacute arterial ischemic infarct in the right middle cerebral artery territory associated with diffuse supratentorial white matter T2-hyperintensity, likely related to radiation-induced leukoencephalopathy. Conversely, the cystic lesions and leptomeningeal enhancement were reduced. In the following weeks, thanks to antiepileptic and rehabilitative treatment the neurological conditions improved and the patient was discharged home. Unfortunately, in the following months, the patient worsened again and underwent a devastating clinical course characterized by an arterial ischemic infarct in the left middle cerebral artery territory (Figure 5), progressive neurological deterioration, consciousness alteration, repetitive seizures, central panhypopituitarism, and need for enteral nutrition. Craniospinal MRI showed no signs of disease progression. Finally, the child died of bronchopneumonia at the age of 9.4 years, 7 years after clinical onset. The parents did not authorize autopsy.

\section{Case 3}

A previously healthy 13-year-old boy presented to another hospital with sudden onset of awakening at night, confusional state, dysarthria, and dyslalia. Dysgraphia and difficulty in concentration in the previous weeks were also reported by the parents. Contrast-enhanced craniospinal MRI depicted some cystic lesions over the cerebellar surface and diffuse leptomeningeal enhancement particularly in the cerebral and cerebellar subarachnoid spaces, pontine and ambient cisterns, and spinal cord, with no evidence of intraparenchymal involvement. Moreover, linear hypointensities on $\mathrm{T} 2$-weighted images were observed along the posteromedial surface of the thalami with corresponding calcifications on CT scan (Figure 4I). A small calcification was retrospectively noted also within the left sylvian fissure. The search of most common infectious markers in the cerebrospinal fluid was negative. A diagnosis of DLGNT was made through a biopsy of the pathological tissue at the spinal level, and a wait-and-see approach was maintained for the first 22 months. At age 14, as described in (25), the boy presented to our Institution due to slight clinical worsening with seizures, character changes (hyperactivity, mood imbalance) and one episode of partial epilepsy; neurological examination remained normal without focal deficits. Craniospinal MRI showed worsening of the neuroimaging features. Therefore, a chemotherapy treatment with vincristine and carboplatin was started according to the international SIOP-LGG protocol. After a few months, due to the onset of neurotoxicity 1 mainly consisting of a peripheral neuropathy, this chemotherapy regimen was interrupted and a second line chemotherapy was started with oral temozolomide (5 cycles) without apparent radiological benefit. Parents thus asked to interrupt chemotherapy. Following the appearance of seizures secondary to the disease, we decided to prescribe valproic acid $(15 \mathrm{mg} / \mathrm{kg}$ twice a day) also for its autophagic and apoptotic mechanism in central nervous system malignancies, with both clinical and radiological improvements after 3 and 6 months. In the following years, during a long and essentially stable clinical course, multiple MRI examinations showed reduction of the leptomeningeal enhancement and progressive appearance of diffuse supra- and infratentorial cortical and leptomeningeal hypointensities on T2* GE images. Conversely, CT scans documented an increase of calcifications only at the level of the leptomeninges over the posteromedial surfaces of the thalami, right frontal and bilateral temporal sulci (Figure $47-4 L)$. At 23 years of age, due to a slow and progressive cognitive decline characterized by spatial and temporal disorientation, attention and memory disorders, together with a slight neuro radiological worsening, a therapy with Vinblastin $\left(6 \mathrm{mg} / \mathrm{m}^{2}\right.$ weekly) was started, but then stopped after only 2 months for further clinical deterioration in terms of walking disorders and cognitive impairment. We thus decided to treat the patient with Bevacizumab $(10 \mathrm{mg} / \mathrm{Kg} /$ dose biweekly). In the first 6 months of treatment, the patient showed a slight neurological improvement with diffuse reduction of leptomeningeal contrast enhancement on MRI followed by radiological stabilization (Figure 3). However, after 20 months, at the age of 25.8 years, the neurological conditions heavily worsened, and, in agreement with parents, we decided to stop the treatment with Bevacizumab. The patient is still alive, at home. 